\title{
Geometry and dynamics of planar linkages
}

\author{
M. L. S. Magalhães *and M. Pollicott ${ }^{\dagger}$
}

November 29, 2011

\begin{abstract}
In this article we present an approach to describing the geometry and curvature of the configuration spaces of a class of simple idealized planar linkages. This is based on determining the curvature of such configuration spaces canonically embedded into Euclidean space, and then the behaviour of the dynamics of such linkages can be understood via the associated geodesic flow. Our objective is to present a method which, in principle, can be applied to many different examples.
\end{abstract}

\section{Introduction}

A mechanical linkage can be viewed as a series of rigid rods where each rod has two either fixed pivots or movable joints connecting them. The origins of their study date back to the classical work of Euler, Watt, Chebyshev and others in the 19th Century [12].

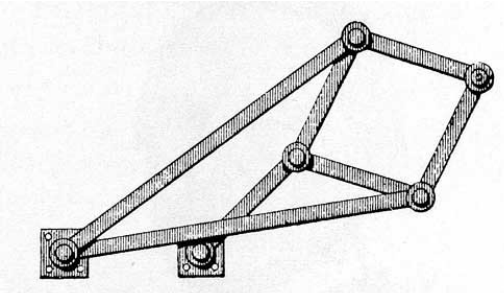

Figure 1: An elegant illustration of the Peaucellier-Lipkin mechanical linkage from Kempe's 1877 book "How to draw a straight line"

In modern times, Thurston studied the problem of which configuration spaces can occur, which inspired Kapovich and Millson to gave a complete proof of the Universality Theorem, essentially saying that for any compact manifold $M$ one can choose a linkage whose phase space contains a component homeomorphic to $M$ [10]. For more on topology of the configuration space, we refer the reader to the book of Farber [6] (and the extended french version of the article of Thurston and Weeks [17]).

${ }^{*}$ M. L. S. Magalhaes, Departmento de Matemática, Universidade do Porto, Porto 4169-007, Portugal Email: mlsmagal@fc.up.pt

${ }^{\dagger}$ Mark Pollicott, Department of Mathematics, Warwick University, CV4 7AL, UK Email: masdbl@warwick.ac.uk 
An interesting new ingredient introduced by Hunt and MacKay [8] was to consider the dynamics of such a mechanical system. To make the analysis more tractable, useful simplifying assumptions are:

1. the joints have non-zero mass;

2. the rods have no mass or moment of inertia; and

3. that the system has no friction.

There is then a canonical embedding of the phase space into Euclidean space $M \subset \mathbb{R}^{2 n}$, say, by the coordinates of the $n$ movable joints in the plane.

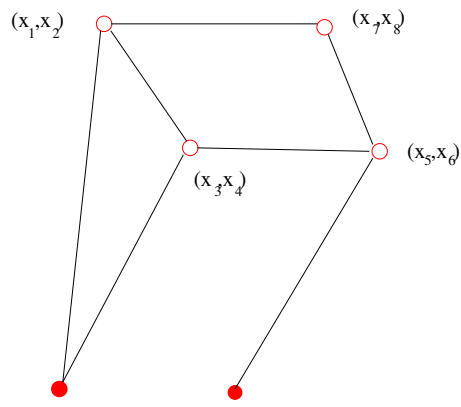

Figure 2: In this figure, each of the four joints contributes a pair of coordinates to the point $\left(x_{1}, x_{2}, \cdots, x_{8}\right) \in M \subset \mathbb{R}^{8}$ in the configuration space.

In particular special cases, there are embeddings into lower dimensional spaces Euclidean spaces which often simplifies the analysis. For example, if a free vertex (joint) is attached to a fixed vertex (pivot) by a rod then rather than considering position of the free vertex in terms of its two dimensional Euclidean coordinates it may be specified more economically by the (one dimensional) angle of the rod. In this context the Euclidean space may be the product of a usual Euclidean space with a flat torus, i.e., $\mathbb{R}^{n} \times \mathbb{T}^{m}$.

The geodesic flow on the embedded manifold with the induced metric describes the dynamics of this idealized linkage. From the point of view of ergodic theory, one might consider the behaviour of typical trajectories of linkages with respect to the Liouville measure, say. From the point of view of topological dynamics, one might consider the behaviour of closed trajectories of linkages, for example.

We can summerize the basic approach as follows.

Step 1: The behaviour of the geodesic flow is determined by the curvature of the configuration space $M$ (embedded into Euclidean space).

Step 2: The dynamics of an idealised mechanical linkage is described by the orbit of the geodesic flow on the configuration space $M$.

Thus, we are naturally brought to the main problem we need to address. 
Question 1.1. Given a linkage with fixed lengths for the rods, how can we compute the curvature of the configuration space, using the metric arising from the canonical embedding into Euclidean space?

\section{Configuration space and Geodesic flows}

We now begin a more detailed description of our analysis.

\subsection{The configuration space}

We begin by presenting a more formal definition of the type of idealised planar linkage we want to consider.

1. We can consider a connected planar graph $\mathcal{G}=(\mathcal{V}, \mathcal{E})$ consisting of a finite set of vertices $\mathcal{V}$ and edges $\mathcal{E}$.

2. We next partition the vertex set into a disjoint union $\mathcal{V}_{\text {fixed }} \cup \mathcal{V}_{\text {free }}=\mathcal{V}$ (where the subscripts refer to what we shall call "fixed" and "free" vertices, respectively).

3. Moreover, we associate to each edge $e \in \mathcal{E}$ a fixed length $l(e) \in \mathbb{R}$.

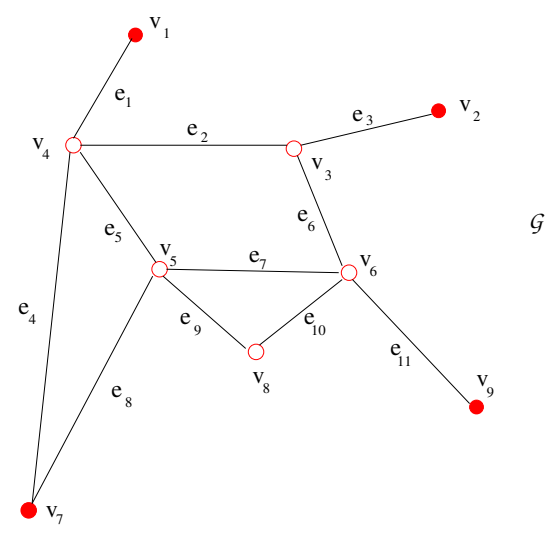

Figure 3: A graph $\mathcal{G}$ with nine vertices (the four solid dots corresponding to fixed pivots and five hollow dots corresponding to movable joints) and eleven edges (corresponding to rods of fixed length)

We can think of $\mathcal{V}_{\text {fixed }}, \mathcal{V}_{\text {free }}$ and $\mathcal{E}$ as representing the fixed pivots, free joints and rods. respectively. In particular, $\mathcal{V}_{\text {free }}$ and $\mathcal{E}$ are free to move in the plane, subject to the edge lengths $l(e), e \in \mathcal{E}$, being preserved. We let $\mathcal{L}=\mathcal{L}\left(\mathcal{V}_{\text {fixed }}, \mathcal{V}_{\text {free }}, l\right)$ denote the space of possible configurations of the associated linkage. 


\subsection{The embedding}

One can naturally map the space of all such linkage configurations $\mathcal{L}=\mathcal{L}\left(\mathcal{V}_{\text {fixed }}, \mathcal{V}_{\text {free }}, l\right)$ into a $\left|\mathcal{V}_{\text {free }}\right|$-dimensional Euclidean space $\left(\mathbb{R}^{2}\right)^{\mid \mathcal{V}_{\text {free }}}$. More precisely, we define the map $\iota: \mathcal{L} \rightarrow\left(\mathbb{R}^{2}\right)^{\left|\mathcal{V}_{\text {free }}\right|}=\mathbb{R}^{2\left|\mathcal{V}_{\text {free }}\right|}$ by

$$
\iota(L)=\left(v_{1}(L), \cdots, v_{\left|\mathcal{V}_{\text {free }}\right|}(L)\right)
$$

where $v_{1}(L), \cdots, v_{\mid V_{\text {free }}}(L) \in \mathbb{R}^{2}$ are the coordinates in the plane of the positions of each of the free vertices of a given linkage $L \in \mathcal{L}$. The image $M=\iota(\mathcal{L}) \subset\left(\mathbb{R}^{2}\right)^{\left|\mathcal{V}_{\text {free }}\right|}$ is defined implicitly by a finite number of equations and thus is clearly an algebraic variety and, perhaps surprisingly, typically an embedded manifold [? ].

We can generalize this basic construction in two particularly useful ways. Firstly, we can change the coordinates on the axes of the embedding space $\left(\mathbb{R}^{2}\right)^{\left|\mathcal{V}_{\text {free }}\right|}$ by fixing scaling factors $m_{1}, \cdots, m_{\left|\mathcal{V}_{\text {free }}\right|}>0$. This then allows us to define a more general map $\iota: \mathcal{L} \rightarrow\left(\mathbb{R}^{2}\right)^{\left|\mathcal{V}_{\text {free }}\right|}=$ $\mathbb{R}^{2\left|\mathcal{V}_{\mid \text {free }}\right|}$ by

$$
\iota(\mathcal{L})=\left(\sqrt{m_{1}} v_{1}(\mathcal{L}), \cdots, \sqrt{m_{\mid V_{\text {free }}} \mid} v_{\mid V_{\text {free }}}(\mathcal{L})\right)
$$

Secondly, if we have $n_{1}$ free joints each of which is connected to a fixed pivot then their positions can be described by a single angle on the circle $\mathbb{T}$ rather than by a two dimensional coordinate in $\mathbb{R}^{2}$. If we still use two dimensional coordinates to record the positions of the remaining $n_{2}:=\left|V_{\text {free }}\right|-n_{1}$ coordinates then we can embedded the configuration in the Euclidean space $\mathbb{T}^{n_{1}} \times \mathbb{R}^{n_{2}}$, again with the metric on $M$ being induced from the standard Euclidean metric. This has the advantage that their is a reduction by $n_{1}$ in the dimension of the ambient space.

\subsection{The geodesic flow}

In the absence of other forces, the dynamics of the linkage $L$ is described entirely by the kinetic energy of the masses on the free vertices, or joints. Let us assume that the vertices $v_{1}(L), \cdots, v_{\left|\mathcal{V}_{\text {free }}\right|}(L) \in \mathcal{V}_{\text {free }}$ have corresponding masses $m_{1}(L), \cdots, m_{\left|V_{\text {free }}\right|}(L)>0$, say. The kinetic energy is then given by

$$
\mathcal{K}_{\text {linkage }}=\frac{1}{2} \sum_{i=1}^{\left|\mathcal{V}_{\text {free }}\right|} m_{i}\left(\frac{v_{i}(L)}{d t}\right)^{2}
$$

Let us consider the embedding of the configuration space given by (2.1). In particular, let $g=\left(g_{i j}\right)$ denote the induced metric on $M$ in local coordinates. The differential equations for the geodesics on $M$ associated to this metric take the familiar form

$$
\frac{d^{2} x^{i}}{d t^{2}}+\sum_{j k} \Gamma_{j k}^{i} \frac{d x^{j}}{d t} \frac{d x^{k}}{d t}=0
$$

where

$$
\Gamma_{j k}^{i}=\frac{1}{2} \sum_{m} \mathrm{~g}^{i m}\left(\frac{\partial \mathrm{g}_{m j}}{\partial x^{k}}+\frac{\partial \mathrm{g}_{m k}}{\partial x_{j}}-\frac{\partial \mathrm{g}_{j k}}{\partial x_{m}}\right)
$$


are the Christoffel symbols. The associated geodesic flow $\phi_{t}: S M \rightarrow S M$ on the unit tangent bundle is defined by parallel transporting unit tangent vectors along these geodesics. In particular, under the canonical projection $\pi: S M \rightarrow M$ the orbits of the geodesic flow project to geodesics on $M$. Finally, the kinetic energy of the geodesic flow is given by

$$
\mathcal{K}_{\text {geodesic }}=\frac{1}{2} \sum_{i=1}^{2\left|\mathcal{V}_{\text {free }}\right|}\left(\frac{d x_{i}}{d t}\right)^{2}
$$

The geodesic flow is an example of a Hamiltonian flow and we can restrict the geodesic flow to constant energy manifolds. In particular, we can choose that $\mathcal{K}_{\text {linkage }}=\mathcal{K}_{\text {geodesic }}$ and this leads us to the following result.

Proposition 2.1 (Hunt-Mackay). The trajectories of a linkage correspond to the orbits of the geodesic flow on $M$ with respect to the induced Euclidean metric.

In terms of the configuration space, this corresponds to the trajectory for the geodesic flow starting at $x$ with initial condition $v \in T V$, say. i.e., we choose a geodesic $\gamma_{v}: \mathbb{R}^{+} \rightarrow V$ with $\gamma_{v}(0)=x$ and $\dot{\gamma}_{v}(0)=v$. Let $\phi_{t}: T_{1} M \rightarrow T_{1} M$ be the geodesic flow on the unit tangent bundle $T_{1} M$ defined by $\phi_{t}(v)=\dot{\gamma}_{v}(t)$.

A knowledge of the sectional curvatures of manifold $M$ is one of the principle tools that can be used in describing the behaviour of geodesic and thus, in the present context, of the evolution of the linkages. To illustrate this principle, let us consider two extreme cases:

1. If we can find a closed geodesic $\gamma$ on the configuration space which lies entirely in a region of positive curvature then this will give rise to a (positive measure) family of geodesics which oscillate in a neigbourbood of $\gamma$ and, in particular, the geodesic flow will not be ergodic with respect to the Liouville measure say.

2. On the other hand, if we find a closed geodesic $\gamma$ on the configuration space which lies in a region of negative curvature then in a neigbourbood of $\gamma$ the dynamics will be seen to be hyperbolic and unstable. In the extreme case that the curvature is negative on a sufficiently large part of the configuration space then the flow is typically Anosov, and thus automatically ergodic with respect to the Louville measure.

Consider the particular case that $M$ is a surface. There are particular constraints on the curvature that a surface may have imposed by its topology and the Gauss-Bonnet theorem. More precisely, the average Gaussian curvature must be equal to the Euler characterisitc and so, in particular, a surface with positive (or negative) Euler characterisitc must necessarily have regions with positive (or negative) curvature. Moreover, there are additional constraints imposed on the possible curvature of surfaces embedded into low dimensional Euclidean spaces. For example, the famous Hilbert Theorem prohibits a closed constant negatively curved surface being embedded into $\mathbb{R}^{3}{ }^{1}$

\footnotetext{
${ }^{1}$ Moreover, any complete surface with negative curvature $\kappa \leq \delta<0$ cannot be embedded in $\mathbb{R}^{3}$ (or any three dimensional Euclidean space) [5] cf. [13]).
} 


\section{$3 \quad$ Periodic trajectories}

We now turn our attention to closed geodesics in the configuration space. These correspond to periodic trajectories of configurations.

We now recall two classical facts from differential geometry.

Lemma 3.1. Let $V$ be a compact surface.

1. Every free homotopy class ${ }^{2}$ contains at least one closed geodesic.

2. If $V$ has strictly negative curvature then each free homotopy class contains at least one closed geodesic.

3. A closed geodesic is locally extremal in length.

A prime periodic trajectory $\tau$ is one for which a configuration $v \in \tau$ is repeated in a finite time, i.e., $v=\dot{\gamma}_{v}(\lambda(\tau))$ for some $\lambda(\tau)>0$, say, and for which $\lambda(\tau)>0$ is least. The following simple result gives the existence of infinitely many periodic trajectories when the configuration space $V$ is a surface.

Proposition 3.2. If the configuration space corresponds to an oriented surface $V$ then there are infinitely many prime periodic trajectories. Moreover, if the configuration space has genus $g \geq 2$ then the number of (hyperbolic) closed trajectories grows exponentially, i.e.,

$$
\limsup _{T \rightarrow \infty} \frac{1}{T} \log \operatorname{Card}\{\tau: \lambda(\tau) \leq T\}>0
$$

Proof. Since the dynamics corresponds to the geodesic flow on the surface $V$, it suffices to prove the corresponding results for closed geodesics on $V$. We consider separately the three cases $g=0, g=1$ and $g \geq 2$.

Assume first that $g=0$. Then we can apply the (solution to) the Klingenberg conjecture, that for any metric on the two sphere there are infinitely many closed geodesics. This was proved by Bangert [1] and Franks [7];

Assume next that $g=1$. Since there are infinitely many free homotopy classes, each containing at least one closed geodesic, the result follows.

Finally, if $g \geq 2$ then we can show that the topological entropy $h(\phi)$ for the geodesic flow is non-zero; We refer the reader to [18] for the definition of topological entropy. We first recall the following definition.

Definition 3.3. We can define the volume entropy of the surface $V$ by

$$
h(V)=\lim _{R \rightarrow+\infty} \frac{1}{R} \log \operatorname{Vol}_{\tilde{V}}\left(B_{\tilde{V}}(x, R)\right)
$$

where $B_{\tilde{V}}(x, R)$ denotes a ball of radius $R>0$ in the universal cover $\tilde{V}$ for $V$, with respect to the lifted Riemannian distance and volume.

\footnotetext{
${ }^{2}$ i.e., a conjugacy class in the fundamental group
} 
The definition is independent of the choice of point $x \in \tilde{V}$, and the limit exists by a sub-additivity argument.

We can now use the following result of Manning [14].

Lemma 3.4 (Manning). The topological entropy of the geodesic flow $\phi_{t}: T_{1} V \rightarrow T_{1} V$ satisfies $h(\phi) \geq h(V)$.

Moreover, it is easy to see from the definition that the volume entropy $h(V)$ is bounded below by

$$
h(V) \geq \frac{I(V)}{d(V)} \log (2 g-1)>0
$$

where $I(V)$ is the injectivity radius and $d(V)$ is the diameter. Thus the entropy $h(\phi)$ for the geodesic flow is strictly positive and the result on exponential growth of closed geodesics follows by Katok's theorem [11]:

Lemma 3.5 (Katok). If for a $C^{2}$ flow $\phi_{t}: T_{1} V \rightarrow T_{1} V h(\phi)>0$ then

$$
\limsup _{T \rightarrow \infty} \frac{1}{T} \log \operatorname{Card}\{\tau: \lambda(\tau) \leq T\}>0 .
$$

Combining lemmas 3.6 and 3.7 completes the proof.

Remark 3.6. If we allow the masses of the edges to be non-zero and introduce inertia then the dynamics is defined by a more general Hamiltonian flow. However, many such Hamiltonian flows are actually geodesic flows on manifolds subject to a reparamerization by the Maupertius Principle.

Remark 3.7. The geodesic flow preserves the Riemannian volume. If we all also knew that the metric entropy was non-zero then by Pesin's theorem there will be an invariant ergodic set of positive measure.

\section{Curvature of embedded surfaces}

The main technical issue we need to address is the computation of the curvature of the embedded manifold $M$. For simplicity, we will restrict ourselves to the particular case that $M$ is a surface. This both simplifies the choice of curvature and the formulae, although working in greater generality would not present any significant problems.

Since the curvature of the embedded surface is a local property we can assume without loss of generality that the surface is embedded in $\mathbb{R}^{n}$, say. In the special case of an explicit embedded surface in three dimensional Euclidean space the computation of the curvature is relatively straightforward. For the more general case of a surface embedded in $\mathbb{R}^{n}$, where $n \geq 3$, there are relatively recent simple formulae due to $\mathrm{Xu}$ and Bajaj [19], which we will describe below.

Assume that $x: V \mapsto \mathbb{R}^{n}$ is any isometric embedding of a surface $V$ into $n$-dimensional Euclidean space. Let us write the components of the embedding as

$$
x(u, v)=\left(x_{1}(u, v), x_{2}(u, v), \cdots, x_{n}(u, v)\right) \in \mathbb{R}^{n},
$$


where $(u, v)$ are local coordinates on the surface $V$. There exists a relatively simple and practical formula for the Gaussian curvature $\kappa(u, v)$ at the point $x(u, v)$ on the embedded surface. More precisely, we can define a $2 \times 2$ matrix

$$
G(u, v)=\left(\begin{array}{cc}
\sum_{i=1}^{n}\left(\frac{\partial x_{i}(u, v)}{\partial u}\right)^{2} & \sum_{i=1}^{n} \frac{\partial x_{i}(u, v)}{\partial u} \frac{\partial x_{i}(u, v)}{\partial v} \\
\sum_{i=1}^{n} \frac{\partial x_{i}(u, v)}{\partial u} \frac{\partial x_{i}(u, v)}{\partial v} & \sum_{i=1}^{n}\left(\frac{\partial x_{i}(u, v)}{\partial v}\right)^{2}
\end{array}\right)
$$

and consider its inverse $H(u, v)=G(u, v)^{-1}$. We next consider the $2 \times n$ matrix given by

$$
M(u, v):=\left(\begin{array}{cccc}
\frac{\partial x_{1}(u, v)}{\partial u} & \frac{\partial x_{2}(u, v)}{\partial u} & \cdots & \frac{\partial x_{n}(u, v)}{\partial u} \\
\frac{\partial x_{1}(u, v)}{\partial v} & \frac{\partial x_{2}(u, v)}{\partial v} & \cdots & \frac{\partial x_{n}(u, v)}{\partial v}
\end{array}\right)
$$

and then we can define a $n \times n$ matrix:

$$
Q(u, v)=I-M(u, v)^{T} H(u, v) M(u, v) .
$$

Finally, we define

$$
\begin{aligned}
t_{11}(u, v) & =\left(\begin{array}{llll}
\frac{\partial^{2} x_{1}(u, v)}{\partial u^{2}} & \frac{\partial^{2} x_{2}(u, v)}{\partial u^{2}} & \ldots & \frac{\partial^{2} x_{n}(u, v)}{\partial u^{2}}
\end{array}\right) \\
t_{12}(u, v)=t_{21}(u, v) & =\left(\begin{array}{llll}
\frac{\partial^{2} x_{1}(u, v)}{\partial u \partial v} & \frac{\partial^{2} x_{2}(u, v)}{\partial u \partial v} & \ldots & \frac{\partial^{2} x_{n}(u, v)}{\partial u \partial v}
\end{array}\right) \\
t_{22}(u, v) & =\left(\begin{array}{llll}
\frac{\partial^{2} x_{1}(u, v)}{\partial v^{2}} & \frac{\partial^{2} x_{2}(u, v)}{\partial v^{2}} & \ldots & \frac{\partial^{2} x_{n}(u, v)}{\partial v^{2}}
\end{array}\right) .
\end{aligned}
$$

We recall the following result.

Proposition 4.1 (Xu and Bajaj, [19]). The Gaussian curvature $\kappa(u, v)$ of the embedded surface at $(u, v)$ takes the form

$$
\kappa(u, v)=\frac{t_{11}(u, v) Q(u, v) t_{22}(u, v)^{T}-t_{12}(u, v) Q(u, v) t_{12}(u, v)^{T}}{\operatorname{det} G(u, v)} \in \mathbb{R} .
$$

Proof. For completeness, we present a proof of this result, which appears to be simpler than the original proof in [19]. It is convenient to begin with a more general setting. Let $M^{n+m}$ be a $(n+m)$-dimensional Riemannian manifold with connection $\bar{\nabla}$ and let $N^{n} \subset M^{m+n}$ be an $n$-dimensional embedded submanifold with inherited connection $\nabla$. Given $p \in N$ we define a bilinear symmetric map $S: T_{p} N \times T_{p} N \longrightarrow\left(T_{p} N\right)^{\perp}$ by

$$
S\left(X_{p}, Y_{p}\right):=\bar{\nabla}_{X_{p}} Y-\nabla_{X_{p}} Y
$$

where $X_{p}, Y_{p} \in T_{p} N, \bar{Y}$ is a local extension of $Y_{p}$ to a vector field $Y$ on $M$ tangent to $N$. We note that $S\left(X_{p}, Y_{p}\right) \in T_{p} M$ is normal to $T_{p} N$ and does not depend on the extension $Y$. We recall the following classical identity.

Lemma 4.2. (Gauss Identity). Let $X, Y, Z, W$ be vector fields on $N$. Then

$$
\langle\bar{R}(X, Y) Z, W\rangle=\langle R(X, Y) Z, W\rangle-\langle S(X, Z), S(Y, W)\rangle+\langle S(Y, Z), S(X, W)\rangle
$$

where $\bar{R}$ and $R$ are respectively the Riemann curvature tensors on $M$ and $N$. 
We consider the specific case $M=\mathbb{R}^{m+n}$. Then the left hand side of equation (4.1) is zero. Moreover, letting $n=2$, the formulae for the (Gaussian) curvature is given by

$$
\kappa_{p}:=\frac{\langle R(X, Y) X, Y\rangle_{p}}{\left\|X_{p}\right\|^{2}\left\|Y_{p}\right\|^{2}\langle X, Y\rangle_{p}^{2}}
$$

where $X_{p}, Y_{p} \in T_{p} N$ are any linearly independent vectors.

Fix a point $p=x(u, v)$. We choose $X=t_{1}$ and $Y=t_{2}$. Then for $i, j \in\{1,2\}$ we have that $\bar{\nabla}_{t_{i}} t_{j}=t_{i j}$ since $M=\mathbb{R}^{m+n}$ is Euclidean.

From the definition of $S$, we can write $S\left(t_{i}, t_{j}\right)=Q\left(t_{i j}\right)$, where $Q$ is an $(n+m) \times(n+m)$ matrix such that $Q(w)$ is the projection onto $\left(T_{p} N\right)^{\perp}$ of $w \in \mathbb{R}^{m+n}=T_{p} M$. Substituting $X=Z=t_{1}$ and $Y=W=t_{2}$ into equation (1) gives that

$$
\begin{aligned}
\langle R(X, Y) X, Y\rangle & =-\left\|Q\left(t_{12}\right)\right\|^{2}+\left\langle Q\left(t_{11}\right), Q\left(t_{22}\right)\right\rangle \\
& =Q\left(t_{11}\right)^{T} Q\left(t_{22}\right)-Q\left(t_{12}\right)^{T} Q\left(t_{12}\right) \\
& =t_{11}^{T} Q t_{22}-t_{12}^{T} Q t_{12}
\end{aligned}
$$

since $Q$ is symmetric and a projection, i.e., $Q^{T}=Q$ and $Q^{2}=Q$. Thus we conclude from (2) that

$$
k(u, v)=\frac{t_{11}^{T} Q t_{22}-t_{12}^{T} Q t_{12}}{\left\|t_{1}\right\|^{2}\left\|t_{2}\right\|^{2}\left\langle t_{1}, t_{2}\right\rangle^{2}}
$$

as required. This completes the proof

\section{Application I: An articulated arm linkage}

To illustrate the application of Proposition 4.1 to computing the curvature of the configuration spaces of linkages we begin with one of the simplest possible examples. This has the merit that the formula for the curvature is simple to compute and present.

Example 5.1 (Articulated arm linkage). We can consider a linkage with two rods of length $e$ and $1-e$, respectively, with $0<e<1$, a single fixed pivot and two movable joints (as in the figure). In particular, if the angles with the horizontal of the two rods are $\theta_{1}, \theta_{2}$ then the positions of the moving joints are

$$
\begin{aligned}
& \left(u_{1}, v_{1}\right)=\left(e \cos \left(\theta_{1}\right), e \sin \left(\theta_{1}\right)\right) \text { and } \\
& \left(u_{2}, v_{2}\right)=\left(e \cos \left(\theta_{1}\right)+(1-e) \cos \left(\theta_{2}\right), e \sin \left(\theta_{1}\right)+(1-e) \sin \left(\theta_{2}\right)\right)
\end{aligned}
$$

It is immediately clear that the configuration space is a two dimensional torus (with parameterization $\left(\theta_{1}, \theta_{2}\right)$ ). There is a natural embedding into $\mathbb{R}^{4}$ of the form

$$
\left(\theta_{1}, \theta_{2}\right) \mapsto\left(u_{1}, v_{1}, u_{2}, v_{2}\right) .
$$

This corresponds to the case that the two movable joints have the same unit mass, say. 

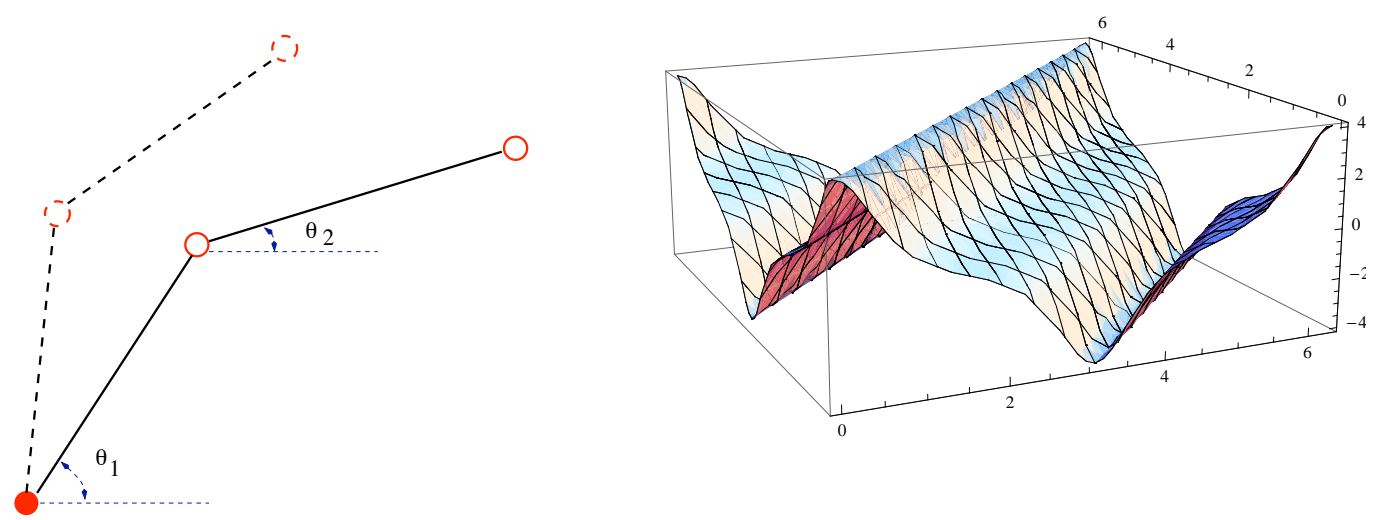

Figure 4: (i) An articulated arm linkage; and (ii) A plot of the curvature for the embedded torus $V$ when $e=\frac{1}{2}$, in terms of the coordinates $0 \leq \theta_{1}, \theta_{2} \leq 2 \pi$.

The next lemma gives an explicit formulae for the the curvature of the embedded configuration space $V$.

Lemma 5.2. The embedded surface $V$ has curvature

$$
\kappa\left(\theta_{1}, \theta_{2}\right)=\frac{4 \cos \left(\theta_{1}-\theta_{2}\right)}{(1-e) e\left(3-\cos \left(2\left(\theta_{1}-\theta_{2}\right)\right)\right)^{2}} .
$$

In particular, we see that the curvature varies between $-\frac{1}{(1-e) e}$ and $\frac{1}{(1-e) e}$.

We now consider the closed geodesics on the embedded surface $V$, which is homeomorphic to a torus $\mathbb{T}^{2}$.

Proposition 5.3. There are two distinguished closed geodesics:

1. $\theta_{1}=\theta_{2}$, which corresponds to a (locally) length minimizing geodesic; and

2. $\theta_{1}=\theta_{2} \pm \pi$, which corresponds to a (locally) length maximizing a geodesic

Proof. By Lemma 5.1 we see that in the first case when $\theta_{1}=\theta_{2}$ then this line has the maximum positive curvature; and when $\theta_{1}=\theta_{2} \pm \pi$ then this line has the minimum negative curvature. Moreover, it is easy to see that the lines corresponding to $\theta_{1}=\theta_{2}$ and $\theta_{1}=\theta_{2} \pm \pi$ are geodesics.

This has the following interpretation for the dynamics of linkages. There are two distinguished cases:

1. $\theta_{1}=\theta_{2}$, which corresponds to those (stable) configurations where the two rods are full extended.

2. $\theta_{1}=\theta_{2} \pm \pi$, which corresponds to those (unstable) configurations where the two rods are doubled up. 

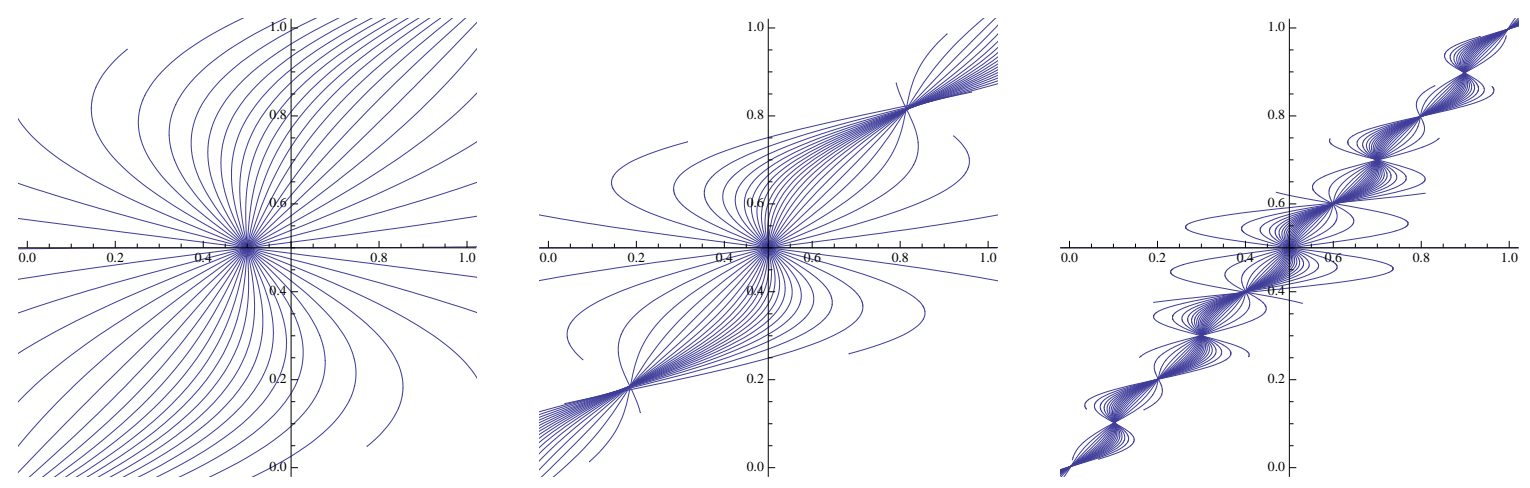

Figure 5: We plot the geodesics emanating from the point $(1 / 2,1 / 2)$ for decreasing $e>0$. The increasing difference in the maximum and minimum curvatures leads to more extreme behavior of the geodesics.

The stability and instability, respectively, of these two geodesics follows from Lemma 3.2. For the corresponding linkage, we can assume that there are unit masses at the movable joints. In particular, we have the following somewhat obvious corollary:

Corollary 5.4. The trajectories in the corresponding configuration space are not transitive (and thus not ergodic).

Remark 5.5. We could have alternatively embedded the configuration space into the three dimensional Euclidean space $\mathbb{T} \times \mathbb{R}^{2}$ using the coordinates $\left(\theta_{1}, u_{2}, v_{2}\right)$.

Remark 5.6. More generally, we could consider a linkage with one fixed pivot and $(n-1)$ free pivots, with $n \geq 3$. Let $\mathcal{V}_{\text {fix }}=\left\{v_{1}\right\}$ and $\mathcal{V}_{\text {free }}=\left\{v_{2}, \ldots, v_{n}\right\}$ with edges $e_{i}=\left(v_{i}, v_{i+1}\right)$ joining $v_{i}$ to $v_{i+1}(i=1, \cdots, n-1)$ of unit length, say, . A convenient configuration space is the $n$-dimensional torus $\mathbb{T}^{n-1}$ where the parameterization is given by the angle each edge $e_{i}$ makes with the horizontal. To study this case we would need to develop a formula for the curvature of $n$-dimensional manifolds embedded in Euclidean space.

\section{Application II: A pentagonal linkage}

We next consider the slightly more complicated example of the pentagonal linkage.

Let $\mathcal{V}_{\text {fix }}=\left\{v_{1}, v_{n}\right\}$ and $\mathcal{V}_{\text {free }}=\left\{v_{2}, \ldots, v_{n-1}\right\}$ with edges $e_{i}=\left(v_{i}, v_{i+1}\right)$ joining $v_{i}$ to $v_{i+1}$ of length $l_{i}$. Let us also denote $l_{0}=\left|v_{1}-v_{n}\right|$. The linkage is precisely a $n$-gon with the edge $\left\{v_{1}, v_{n}\right\}$ fixed. We will concentrate on the case that $n=5$.

The configuration space is two dimensional and is described by the following simple result. We recall that the topology of a surface is described by its first Betti number (i.e., the dimension of the first homology group).

Proposition 6.1. Assume that $\sum_{i} \pm l_{i} \neq 0$ for all possible choices of signs.

1. The configuration space for the pentagon linkage is an (orientable) closed surface.

2. The possible (first) Betti numbers of the surfaces are: 0, 2, 4, 6 or 8 . 


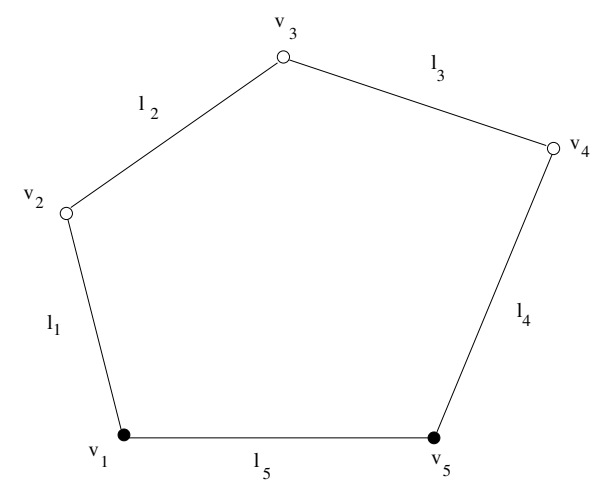

Figure 6: A pentagonal linkage, i.e., a polygonal linkage with $n=5$

We refer the reader to the book of Farber for more details [6] . As a simplification, we can assume that the lengths are symmetric, i.e., $l_{1}=l_{4}$ and $l_{2}=l_{3}$ and $l_{5}=1$.

The following version is easily proved.

Proposition 6.2. Assume without loss of generality that $l_{1}<l_{2}$. If $l_{2}<1$ then we have the following:

1. If $l_{1}+l_{2}<\frac{1}{2}$ then the configuration space is empty since the two ended arms cannot meet at any point $(u, v)$.

2. If $l_{2}>\frac{1}{2}, 0<l_{1}<\frac{1}{2}$ and $\frac{1}{2}<l_{2}-l_{1}$ then $(u, v)$ is restricted to one side and the configuration space consists of a disjoint union of tori, since $\left(\theta_{1}, \theta_{2}\right)$ describes a torus.

3. If $l_{2}>\frac{1}{2}, l_{1}<\frac{1}{2}$ and $l_{2}-l_{1}<\frac{1}{2}$ then the two tori should fuse to become a surface of genus two.

4. If $l_{2}>\frac{1}{2}$ and $l_{1}>\frac{1}{2}$ then the surface has genus 4 .

5. If $l_{2}-l_{1}=\frac{1}{2}$ then the configuration space is singular, being the union of two punctured tori joined at the puncture.

The coordinates of the two free vertices attached by rods to the fixed pivots $(0,0)$ and $(1,0)$ at angles $\theta_{1}, \theta_{2}$ to the horizontal take the form $\left(l_{1} \cos \left(\theta_{1}\right), l_{1} \sin \left(\theta_{1}\right)\right),\left(1+l_{1} \cos \left(\theta_{2}\right), l_{2} \sin \left(\theta_{2}\right)\right)$. The coordinates of the remaining free vertex $(u, v)$ are easily calculated using trigonometry. Given the angles $0<\theta_{1}, \theta_{2}<1$ there are two choices for $(u, v)$, above or below the line joining the first two vertices. This gives a natural involution on the configuration space. This involution need not be an isometry in general.

The configuration space has a natural embedding into four dimensional Euclidean space given by $\left(\theta_{1}, \theta_{2}\right) \mapsto\left(u, v, \theta_{1}, \theta_{2}\right)$. Let us consider the geometry of the embedding for a concrete example.

Example 6.3. With the specific choice $l_{2}=1$ and $0<l_{1}<\frac{1}{2}$ we see from Proposition 6.2 that the configuration space is the torus $\mathbb{T}^{2}$. The explicit formula for the curvature is fairly complicated, but we present three plots for the choices $l_{1}=\frac{1}{5}, \frac{7}{20}$ and $\frac{7}{20}$. 

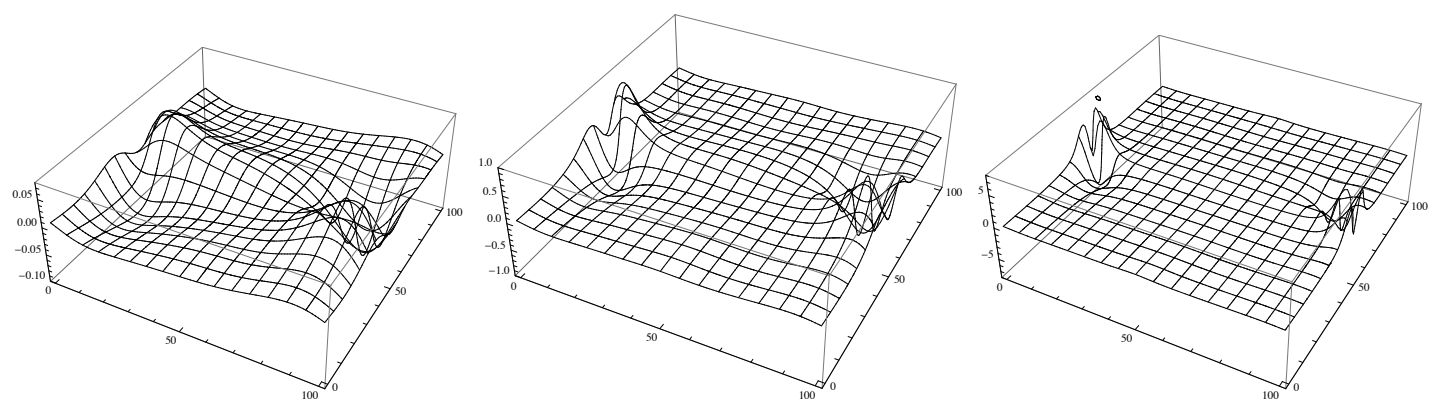

Figure 7: The curvature of the configuration space with $l_{2}=1$ and (i) $l_{1}=\frac{1}{5}$; (ii) $l_{1}=\frac{7}{20}$ and (iii) $l_{1}=\frac{9}{20}$

\section{A further simplification: Asymptotic linkages}

For more elaborate linkages the explicit computation of the curvature proves to be quite complicated. One approach to simplifying the explicit formulae is to allow some of the rods to shrink to an infinitesimally small length $\epsilon$ and then to consider the associated asymptotic configuration space.

Definition 7.1. We denote by $\frac{1}{\epsilon} g_{\epsilon}$ the homothetic scaling of the metric $g_{\epsilon}$ by $\frac{1}{\epsilon} .{ }^{3}$ We say that $g$ is the asymptotic metric for $g_{\epsilon}$ if we have pointwise convergence

$$
\lim _{\epsilon \rightarrow 0} \frac{1}{\epsilon} g_{\epsilon}=g .
$$

In particular, if $\epsilon$ is sufficiently small then the geometry, and in particular the curvature, of $\frac{1}{\epsilon} g_{\epsilon}$ is arbitrarily close to that $g$.

Example 7.2 (Asymptotic pentagon linkage). We can consider the pentagonal linkage consisting of: two short rods of length $\epsilon$ connected to the fixed pivots at $(0,0)$ and $(1,0)$, and at the other ends to long rods of length $\left(\frac{1}{2}+b \epsilon\right)$, for some fixed value of $0<b<1$. Finally, the other ends of the long rods are joined at a common movable pivot $(u, v)$.

If the short rods make angles $\theta_{1}, \theta_{2}$ to the horizontal axis then we can consider the embedding into $\mathbb{T}^{2} \times \mathbb{R}^{2}$ given by

$$
\left(\theta_{1}, \theta_{2}\right) \mapsto\left(\theta_{1}, \theta_{2}, u, v\right) .
$$

The configuration space is a surface of genus 2 and corresponds to two of the pivots having unit mass and the third having the comparatively larger mass $\epsilon^{-1 / 2}$. For a fixed $0<b \leq 1$ we can consider the asymptotic formula as $\epsilon \rightarrow 0$

Proposition 7.3. The asymptotic formula for the curvature as $\epsilon \rightarrow 0$ is:

$$
\begin{aligned}
& \kappa\left(\theta_{1}, \theta_{2}\right)= \\
& \epsilon\left(\frac{\left.\left.-4 b \cos \theta_{1} \cos \theta_{2}-2 \cos ^{2} \theta_{1} \cos \theta_{2}+2 \cos \theta_{1} \cos ^{2} \theta_{2}-\cos \theta_{2} \sin ^{2} \theta_{1}+\cos \theta_{1} \sin ^{2} \theta_{2}\right)\right)}{16\left(2 b+\cos \theta_{1}-\cos \theta_{2}\right)^{2}}\right)+o\left(\epsilon^{3 / 2}\right) .
\end{aligned}
$$

Proof. This follows from the formulae in the previous section by explicit computation. 

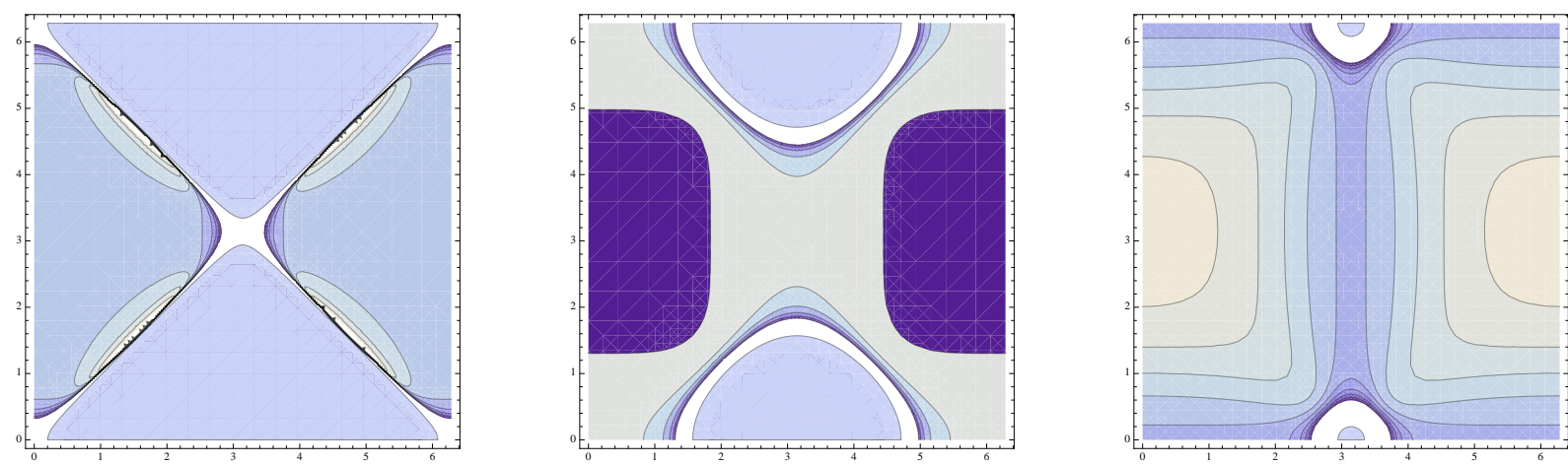

Figure 8: Contour plots of the asymptotic curvature $\kappa\left(\theta_{1}, \theta_{2}\right)$ when (i) $b=0.01$; (ii) $b=0.5$; and (iii) $b=0.99$. The configuration space consists of two copies of the torus attached after the blue disk corresponding to $2 b+\cos \theta_{1}-\cos \theta_{2}<0$ is excised

This allows us to deduce the following:

Proposition 7.4. In the limit $\epsilon \rightarrow 0$ the curvature $\kappa\left(\theta_{1}, \theta_{2}\right)$ converges pointwise provided $2 b+\cos \theta_{1}-\cos \theta_{2}>0$.

We can next consider the limiting case where $b=1$.
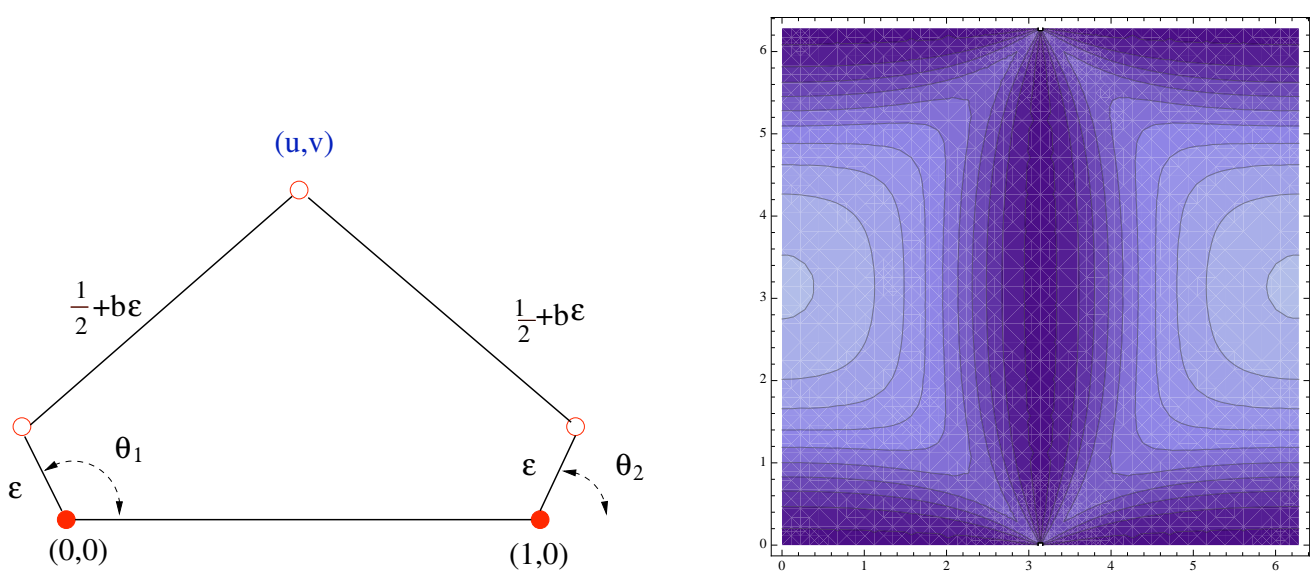

Figure 9: (i) The asymptotic pentagon; and (ii) a plot of the asymptotic curvature $\kappa\left(\theta_{1}, \theta_{2}\right)$ when $b=1$.

Example 7.5 (Asymptotic pentagon linkage with $b=1$ ). In this case, the curvature is for a surface which has a singularity at $\left(\theta_{1}, \theta_{2}\right)=(\pi, 0)=(\pi, 2 \pi)$, but for which otherwise the curvature $\kappa\left(\theta_{1}, \theta_{2}\right)$ converges as $\epsilon \rightarrow 0$. Moreover, the asymptotic formula for the curvature as $\epsilon \rightarrow 0$ simplifies to

$$
\kappa\left(\theta_{1}, \theta_{2}\right)=\epsilon\left(\frac{\left(\cos \theta_{1}-\cos \theta_{2}\right)-\cos \theta_{1} \cos \theta_{2}\left(4 \cos \theta_{1}-\cos \theta_{2}\right)}{16\left(2 b+\cos \theta_{1}-\cos \theta_{2}\right)^{2}}\right)+o\left(\epsilon^{3 / 2}\right) .
$$

\footnotetext{
${ }^{3}$ Recall that metrics $g_{\epsilon}$ are $C^{\infty}$ sections in $\Gamma^{\infty}(V, G L(2, \mathbb{R}))_{+}$taking values in positive definite matrices $G L(2, \mathbb{R})_{+}$.
} 
Remark 7.6. For $b$ previously chosen sufficiently close to 1, the associated surface is smooth, but the curvatures can be arranged close to those in the case $b=1$ (away from the small region $\left.2 b+\cos \theta_{1}-\cos \theta_{2}>0\right)$.

Example 7.7 (Asymptotic spider linkages). Finally, we can consider asymptotic versions of a popular class of examples of linkages, called spider linkages. For $n \geq 2$,

1. Let $\mathcal{V}_{f i x}=\left\{v_{1}, v_{2}, \cdots, v_{n}\right\}$ and $\mathcal{V}_{\text {free }}=\left\{c, w_{1}, w_{2}, \cdots, w_{n}\right\}$;

2. The fixed pivots in $\mathcal{V}_{\text {fix }}$ are the equi-spaced on the unit circle with coordinates

$$
\{(\cos (2 \pi k / n), \sin (2 \pi k / n)): k=0, \cdots, n-1\}
$$

3. Assume that there are edges (or rods) $e_{i}=\left(v_{i}, w_{i}\right)$ joining $v_{i}$ to $w_{i}$ and edges (or rods) $f_{i}=\left(w_{i}, c\right)$ joining $w_{i}$ to $c$ for $i=1, \cdots, n$; and

4. Fix $0<b \leq 1$. Assume that the rods have lengths $l\left(e_{i}\right)=\epsilon$ and $l\left(f_{i}\right)=1+b \epsilon$, for $i=1, \cdots, n$.
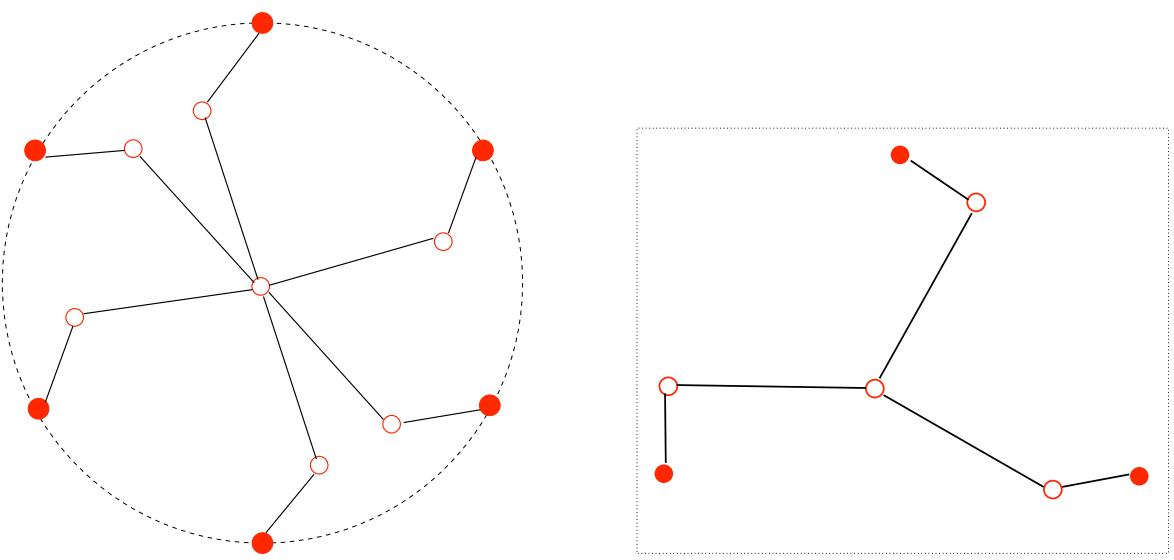

Figure 10: (i) An example of a spider linkage, when $n=6$. The solid dots represent fixed pivots on the unit circle and hollow dots represent freely moving pivots. (ii) When $n=3$ the spider linkage reduces to the Thurston-Weeks triple linkage

Remark 7.8. When $n=3$ this reduces to the Thurston-Weeks triple linkage.

We consider independently each articulated arm linkage attached to a point $(\cos \phi, \sin \phi) \in$ $V_{\text {fix }}$, say, on the unit circle, with angle $\theta$ from the radial lines of the unit circle. By a simple computation, we have the following.

Lemma 7.9. 1. Let $(\epsilon u, \epsilon v)$ be the coordinates of the centre $c$ then

$$
\cos \theta=-b-u \cos \phi-v \sin \phi+O(\epsilon)
$$

2. In particular, for sufficiently small $\epsilon>0$ the configuration space $\left(V, g_{\epsilon}\right)$ will be made up of the union of $2^{n}$ copies of the (small) region consisting of $(u, v)$ which lie the intersection of thin annuli with centre at $(\cos \phi, \sin \phi) \in V_{\text {fix }}$ and having radii $1+(b+1) \epsilon$ and $1+(b-1) \epsilon$. 
3. Asymptotically, as $\epsilon \rightarrow 0$, the points $(u, v)$ lie in strips given by

$$
-b-1 \leq\langle(\cos \phi \sin \phi),(u, v)\rangle=u \cos \phi+v \sin \phi \leq-b+1 .
$$

for all $(\cos \phi \sin \phi) \in V_{f i x}$.

We can use the asymptotic limiting case of (6.1) with $\cos \theta=-b-u \cos \phi-v \sin \phi$ and for $\theta=\theta(u, v)$ to explicitly write the curvature in terms of the angles $\left(\theta_{1}, \cdots, \theta_{n}\right)$.

Remark 7.10. In the particular case that $n=3$ we recover

$$
\kappa=-\frac{3}{2}\left(\frac{\frac{\cos \left(\theta_{1}\right)}{1-\cos ^{2}\left(\theta_{1}\right)} \frac{\cos \left(\theta_{2}\right)}{1-\cos ^{2}\left(\theta_{2}\right)}+\frac{\cos \left(\theta_{2}\right)}{1-\cos ^{2}\left(\theta_{2}\right)} \frac{\cos \left(\theta_{3}\right)}{1-\cos ^{2}\left(\theta_{3}\right)}+\frac{\cos \left(\theta_{3}\right)}{1-\cos ^{2}\left(\theta_{3}\right)} \frac{\cos \left(\theta_{1}\right.}{1-\cos ^{2}\left(\theta_{1}\right)}}{3-\cos \left(2 \theta_{1}\right)-\cos \left(2 \theta_{2}\right)-\cos \left(2 \theta_{3}\right)}\right) .
$$

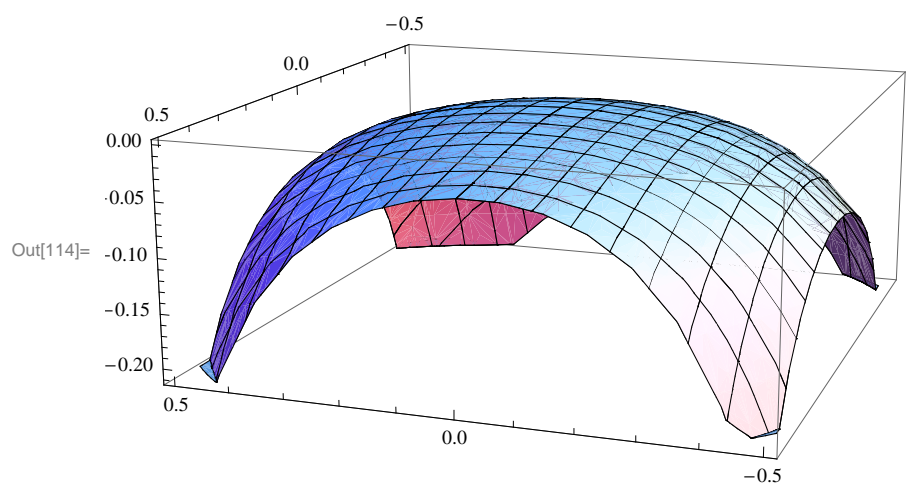

Figure 11: A plot of the curvature of the asymptotic Thurston-Weeks example with $b=0.01$ as a function of $(u, v)$.

We can similarly plot the curvature in terms of $(u, v)$ for other $n$-legged spiders. We exclude the case $n=4$ where the configuration space is again a torus, and which by the Gauss-Bonnet theorem requires the average curvature to be zero.
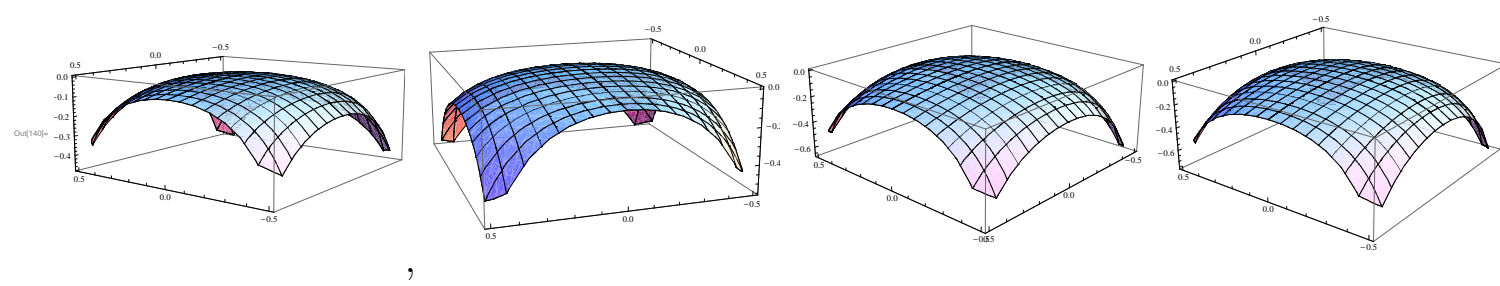

Figure 12: A plot of the curvature of the asymptotic $n$-legged spider linkage when $b=0.01$ and $n=5,6,7,8$, as a function of $(u, v)$. 


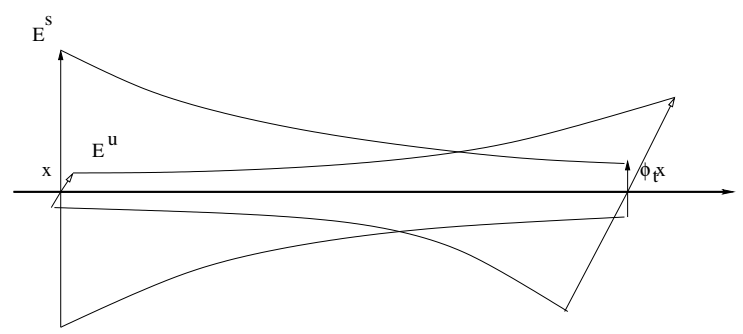

Figure 13: The hyperbolicity transverse to the orbit of an Anosov flow.

\section{Anosov-type geodesic flows}

We now consider one of the most useful properties.

Definition 8.1. We say that $\phi_{t}: T_{1} M \rightarrow T_{1} M$ is of Anosov type if there is a $D \phi_{t}$-invariant Whitney splitting $T\left(T_{1} M\right)=E^{0} \oplus E^{s} \oplus E^{u}$ such that

1. $E^{0}$ is one dimensional and tangent to the flow direction;

2. $\exists C, \lambda>0$ such that $\left\|D \phi_{t} \mid E^{s}\right\| \leq C e^{-\lambda t}$ and $\left\|D \phi_{-t} \mid E^{u}\right\| \leq C e^{\lambda t}$ for $t>0$.

If the manifold is $n$-dimensional then the bundles $E^{s}, E^{u}$ are $(n-1)$-dimensional bundles and the bundle $E^{0}$ is one dimensional. In the particular case that the sectional curvatures are strictly negative at every point on $M$ then the geodesic flow $\phi_{t}: T_{1} M \rightarrow T_{1} M$ is of Anosov type.

The standard method of proof can be adapted to give a criteria for a geodesic flow to be of Anosov type on a surface which has regions of positive curvature (although, of course, we cannot expect the property to hold if, say, there is any closed trajectory that remains in a region of positive curvature).

The key to establishing hyperbolic behaviour is to show that the cone field consisting of Jacobi fields $J(t)$ along geodesics $\gamma(t)$ satisfying $u(t):=J^{\prime}(t) / J(t) \geq 0$ is mapped strictly inside itself. (In particular, if $u(0)=0$ then $u(t)>0$ for some $t>0$.) The Ricatti equation reformulated in terms of $u(t)$ now takes the form

$$
\dot{u}+u^{2}+K(t)=0 .
$$

We are interested in the following property, which is central to showing that the geodesic flow is of Anosov type.

Cone condition: if $u(0) \geq 0$ then there exists $\epsilon>0$ and $t_{0}>0$ such that $u(t) \geq \epsilon$ for $t \geq t_{0}$.

If a closed geodesic has the property that the solution of the equation becomes positive then the geodesic is hyperbolic.

Proposition 8.2. If the cone condition holds then the flow is of Anosov type.

Proof. This is a standard approach, particularly in the special case of surfaces with negative curvature at every point. In particular, it is well known from the classical work of Anosov 
that the negative curvature ensures that the standard cone family $\mathcal{C}^{u}=\left\{J: J \cdot J^{\prime}\right\} \geq 0$ is uniformly contracting, since if the curvature $K \leq-b^{2}<0$, then along a complete geodesic

$$
\frac{d}{d t}\left(J(t) J^{\prime}(t)\right)=J(t) J^{\prime \prime}(t)+J^{\prime}(t)^{2}=-k(t) J(t)^{2}+J^{\prime}(t)^{2} \geq b^{2} J(t)^{2}+J^{\prime}(t)^{2}>0,
$$

unless $J \equiv 0$. It follows that

$$
\frac{d}{d t}\left(J(t) J^{\prime}(t)\right) \geq b^{2} J(t)^{2}+J^{\prime}(t)^{2}>2 b\left(J(t) J^{\prime}(t)\right)
$$

(since the arithmetic mean is larger than the geometric mean). Since $J(1) J^{\prime}(1)>0$ for any nontrivial $J \in \mathcal{C}^{u}$, it follows that $J(t) J^{\prime}(t)$ grows exponentially, and hence $J(t)^{2}+J^{\prime \prime}(t)^{2}$ grows exponentially, since $J^{2}(t)+J^{\prime}(t)^{2} \geq 2 J(t) J^{\prime}(t)$. This is sufficient to establish the Anosov property ${ }^{4}$

In practice, the cone condition can be verified empirically using computer experiments (or laborious calculations by hand).

Example 8.3 (Asymptotic pentagon when $b=1$ ). The curvature is maximized at $\left(\theta_{1}, \theta_{2}\right)=$ $(0, \pi)$ by the value $\frac{1}{32}$ and the positive curvature is in a disk (on each of the two tori). The curvature is minimized at $\left(\theta_{1}, \theta_{2}\right)=(\pi, \pi)$ by the value $-\frac{1}{16}$.

One can check experimentally that the cone property holds (for b close to 1 and $\epsilon$ close to 0). To begin, we can consider the close geodesic corresponding to $\theta_{2}=\pi$. Heuristically,
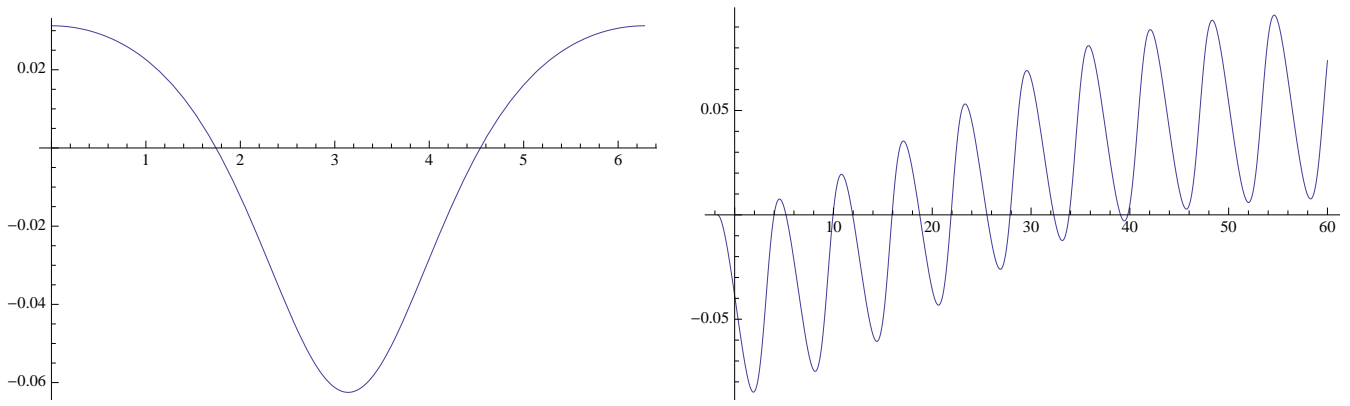

Figure 14: A plot of (i) the curvature $\kappa\left(\theta_{1}, \theta_{2}\right)$; and $u(t)$ along the geodesic corresponding to $\theta_{2}=\pi$, when $b=1$.

this is the geodesic which sees less negative curvature and more positive curvature than the others, and so the validity of the cone property in this case suggests it is true for all other geodesics.

Next we can consider the closed geodesic corresponding to $\theta_{2}=\pi$.

More generally, we can consider geodesics starting from the curve corresponding to zero curvature, i.e., $C=\left\{\left(\theta_{1}, \theta_{2}\right): \kappa\left(\theta_{1}, \theta_{2}\right)=0\right\}$. We can consider those geodesics directed into the region of positive curvature then the solution Ricatti equation with initial value zero initially becomes negative. However, once the geodesic traverses the disk of positive

\footnotetext{
${ }^{4}$ The unstable direction at $v$ corresponds to the intersection $\cap_{t \geq 0} D \phi_{t} \mathcal{C}^{u}\left(\phi_{-t} v\right)$ of the images $D \phi_{t} \mathcal{C}^{u}\left(\phi_{-t} v\right)$ of the cones $C^{u}\left(\phi_{-t} v\right)$ above $\phi_{-t} v$.
} 

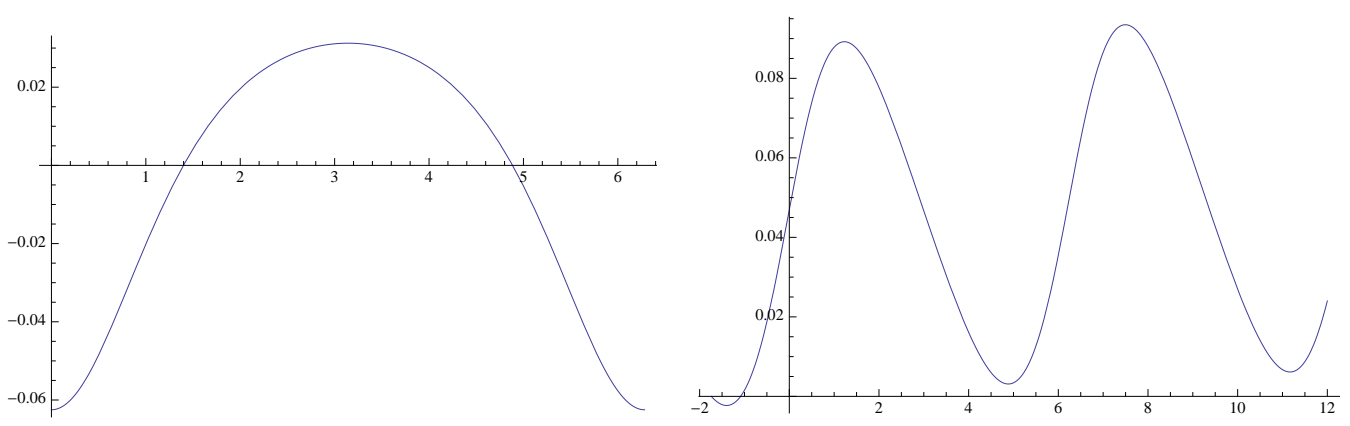

Figure 15: A plot of (i) the curvature $\kappa\left(\theta_{1}, \theta_{2}\right)$; and $u(t)$ along the geodesic corresponding to $\theta_{1}=0$, when $b=1$.

curvature and re-enters the region of negative curvature (by crossing $C$ a second time) the solution begins to increase again. Finally, it suffices to check that the solution becomes strictly positive by the time it re-enters the region of positive curvature, by crossing $C$ a third time, assuming this happens.

To check the hypotheses empirically, we can choose evenly distributed points on $C$ and inwardly pointing vectors.

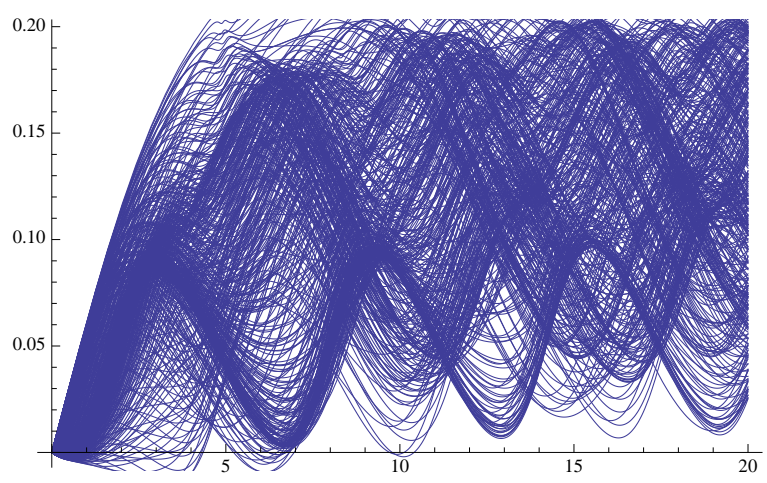

Figure 16: The superposition of the plots of the solutions to the Ricatti equation (in the case $b=1$ ) for 80 points on $C$, each with 20 inwardly directed vectors. The cone condition corresponds to all the solutions eventually becoming positive

\section{$9 \quad$ Ergodic Properties}

The Anosov property of the geodesic flow on the configuration space automatically leads to a number of standard results. Let $\mu$ denote the normalized Liouville measure then by the Birkhoff ergodic theorem for any continuous function $F: T_{1} M \rightarrow \mathbb{R}$ we have that

$$
\lim _{T \rightarrow+\infty} \frac{1}{T} \int_{0}^{T} F\left(\phi_{t} v\right) d t=\int F d \mu
$$

for a.e. $(\mu) v \in T_{1} M$, cf. [2]. Moreover, the Anosov property leads to stronger results. 
Theorem 9.1. For the Anosov flow we have:

1. For any $\epsilon>0$ we have that

$$
\frac{1}{T} \int_{0}^{T} F\left(\phi_{t} v\right) d t=\int F d \mu\left(1+O\left(T^{\frac{1}{2}-\epsilon}\right)\right) \text { as } T \rightarrow+\infty
$$

\section{(Error term in Birkhoff Ergodic Theorem);}

2. For any smooth function $F: S V \rightarrow \mathbb{R}$ we have that there exists $\epsilon>0$ such that

$$
\int F \circ \phi_{t} F d \mu=\left(\int F d \mu\right)^{2}\left(1+O\left(e^{-\epsilon t}\right)\right) \text { for } t>0
$$

\section{(Exponential Mixing);}

3. There exists $\sigma>0$ such that for any $a<b$ we have that

$$
\mu\left\{v: \frac{1}{\sqrt{T}} \int_{0}^{T} F\left(\phi_{t} v\right) d t-\int F d \mu \in[a, b]\right\} \rightarrow \frac{1}{\sqrt{2 \pi} \sigma} \int_{a}^{b} e^{-u^{2} /\left(2 \sigma^{2}\right)} d t \text { as } T \rightarrow+\infty,
$$

\section{(Central Limit Theorem).}

Proof. These results for Anosov flows can be found in [9], [16], [4], [3].

There are also error terms in the convergence in part 3 of Theorem 9.1, corresponding to the classical Berry-Essen theorem. It is also possible to prove even stronger distributional results on individual orbits. These include the Functional Central Limit theorem (or weak invariance principles) and the strong invariance principles, which generalize the Central Limit Theorem.

Definition 9.2. Given any non-trivial non-zero closed 1 -form $w$ and $T>0$ can associate a winding number

$$
c(v, T):=\int_{0}^{T}\langle\omega, X\rangle\left(\phi_{t} v\right) d t \in \mathbb{R} .
$$

This gives some measurement of the displacement of the orbit in a direction in homology determined by $\omega$. For example, we can choose $\omega$ to count the number of times a rod winds around a pivot.

For any $T>0$, we can consider the proportion $\rho_{v}^{+}(T)$ of time $0 \leq t \leq T$ that $c(v, T) \geq 0$. The following is a consequence of the invariance principles.

Theorem 9.3. For any $0<\alpha<1$ we have that

$$
\lim _{T \rightarrow+\infty} \mu\left\{v \in T_{1} V: \frac{\rho_{v}^{+}(T)}{T} \geq \alpha\right\}=\frac{2}{\pi} \sin ^{-1}(\sqrt{\alpha}) .
$$

Similarly, we can consider the proportion $\rho_{v}^{-}(T)$ of time $0 \leq t \leq T$ that $c(v, T) \leq 0$. There is a corresponding result for $\rho_{v}^{-}(T)$. 
Remark 9.4. This leads to some famously counter intuitive results. For example, the probability that a typical geodesic spends a proportion of its time greater than $\frac{3}{4}$ with $c(v, t) \geq 0$ is $\frac{1}{3}$.

Finally, he basic growth rate of close trajectories is equal to the topological entropy. However, one can apply a standard result of Margulis [15] on closed geodesics to prove the following asymptotic formula improving on Lemma 3.5.

Theorem 9.5. Let $N(T)$ denote the number of closed trajectories of period at most $T$, and let $N_{0}(T)$ denote the number of closed trajectories of period at most $T$ such that the trajectory has zero total winding around a given pivot. Then

1. $\lim _{T \rightarrow+\infty} \frac{N(T)}{e^{h T} / T}=h ;$ and

2. $\lim _{T \rightarrow+\infty} \frac{N(T)}{e^{h T} / T^{3 / 2}}=C$, for some constant $C>0$.

\section{References}

[1] V. Bangert, On the existence of closed geodesics on two-spheres, Internat. J. Math. 4 (1993), no. 1, 1-10

[2] I.P. Confeld, S.P. Fomin and Ya. G. Sinai, Ergodic Theory, Springer, Berlin, 1990

[3] M. Denker and W. Philipp, Approximation by Brownian motion for Gibbs measures and flows under a function, Ergod. Th. and Dynam. Sys. 4 (1984), no. 4, 541552.

[4] D. Dolgopyat, On decay of correlations in Anosov flows, Ann. of Math., 147 (1998), no. 2,357390 .

[5] N. V. Efimov, Impossibility a complete regular surface in Euclidean 3-space whose Gaussian curvature has a negative upper bound, Sov. Math. Dokl. 4 (1963), no.1, 843-846.

[6] M .Farber, Invitation to Topological Robotics, European Mathematical Society, Zurich, 2008

[7] J. Franks, Geodesics on $S^{2}$ and periodic points of annulus homeomorphisms, Invent. Math. 108 (1992), no. 2, 403-418.

[8] T.J. Hunt and R. S. MacKay Anosov parameter values for the triple linkage and a physical system with a uniformly chaotic attractor, Nonlinearity 16 (2003) 1499-1510.

[9] A. G., Kachurovskii, Rates of convergence in ergodic theorems, Russian Math. Surveys 51 (1996), no. 4, 653703

[10] M. Kapovich and J. Millson, Universality theorems for configuration spaces of planar linkages, Topology 41 (2002), no6, 1051-1107.

[11] A. Katok, Lyapunov exponents, entropy and periodic orbits for diffeomorphisms, Inst. Hautes tudes Sci. Publ. Math. No. 51 (1980), 137-173. 
[12] A. B. Kempe, How to draw a straight line ; a lecture on linkages, McMillan, London, 1977. (cf. http://www.archive.org/details/howtodrawstraigh00kemprich)

[13] T. Klotz Milnor, Efimov's theorem about complete immersed surfaces of negative curvature, Advances in Mathematics, 8 (1972) 474-543

[14] A. Manning, Topological entropy for geodesic flows, Ann. of Math. (2) 110 (1979), no. $3,567-573$.

[15] G. Margulis, Certain applications of ergodic theory to the investigation of manifolds of negative curvature, Functional Anal. Appl. 3 (1969), no. 4, 335-336

[16] M. Ratner, The central limit theorem for geodesic flows on nn-dimensional manifolds of negative curvature, Israel J. Math. 16 (1973), 181197.

[17] W. Thurston and J. Weeks The Mathematics of three dimensional manifolds Scientific American, 251(1984) 94-106

[18] P. Walters, Am introduction to ergodic theory, Graduate Texts in Mathematics 79, Springer, Berline, 1982

[19] G. Xu and C. Bajaj Curvature computations of 2-manifolds in $\mathbb{R}^{k}$, J. Comput. Math. 21 (2003) 681-688 